\title{
La educación superior, un reto para las universida- des comunitarias interculturales en América Latina
}

\author{
Higher Education, a challenge for Intercultural Community Universities in Latin America
}

\author{
Consuelo Lizeth Blandón Jirón
}

\section{Resumen}

En la primera sección se encontrarán con un breve recorrido por el contexto de la educación superior en América Latina, enfatizando en leyes, normas, convenios, constitución política de países que avalan y reconocen a los pueblos indígenas y afrodescendientes. En la segunda sección hace un breve recorrido por algunas universidades comunitarias interculturales de América Latina que han sido fundadas con un solo fin, rescatar los conocimientos endógenos, preparar profesionalmente a los pueblos originarios. La tercera sección, basada en el debate actual de la interculturalidad, la colaboración intercultural en investigación, un aporte en conjunto con las comunidades, donde se pone en relieve la forma de colaborar desde la investigación y la vinculación existente para que las comunidades indígenas y afrodescendientes puedan gozar de un mejor plan de vida, dar respuestas a problemas que pueden ser resueltos desde la investigación-comunidad.

Palabras Clave: Educación Superior; Interculturalidad; Universitades Comunitarias; Pueblos originarios.

\section{Abstract}

In the first section you will find a brief tour of the context of higher education in Latin America, emphasizing laws, regulations, conventions, political constitution of countries that endorse and recognize indigenous and Afro-descendant peoples. In the second section, a brief tour of some intercultural community universities in Latin America that have been founded with a single purpose it is covered, to rescue endogenous knowledge, to professionally prepare native peoples. The third section, based on the current debate on interculturality, intercultural collaboration in research, a joint contribution with communities, where the way of collaborating from research and the existing linkage for indigenous and Afro-descendant communities is highlighted, so they can enjoy a better life plan, give answers to problems that can be solved from community research.

Keywords: Higher Education; Interculturality; Community Universities; originative peoples.

\section{Introducción}

En este documento basado en un recorrido histórico y en los retos que enfrenta actualmente la educación superior con el tema de la interculturalidad; un término nuevo, en construcción, en debate; pero con muchos desafíos actualmente. La interculturalidad apunta a ese escenario de respeto, tolerancia, diversidad, diálogo horizontal, aceptación mutua para el establecimiento de sociedades inclusivas, por ende, tomando en cuenta a los pueblos originarios. Las Instituciones de Educación Superior (IES) en América Latina, tienen retos actuales para una educación intercultural, la generación de nuevos conocimientos,

\footnotetext{
1 Máster en Docencia Universitaria. Secretaria Académica de la Universidad de las Regiones Autónomas de la Costa Caribe Nicaragüense-Recinto Universitario Nueva Guinea. Email: consuelouraccan@gmail.com, ORCID: https://orcid.org/0000-0003-0301-489X
} 
formación de profesionales integrales, la inclusión de diferentes saberes ancestrales vinculados a la investigación- comunidad.

Actualmente existen universidades comunitarias e interculturales en América Latina como: La Universidad de las Regiones Autónomas de la Costa Caribe Nicaragüense (URACCAN), Universidad Autónoma Indígena Intercultural (UAIIN), Colombia, Universidad Intercultural de las Nacionalidades y Pueblos Indígenas (AMAWTAY WASI), Ecuador, Universidades de Bolivia (UNIBOL). Todas nacen y se establecen para responder a las necesidades que poseen los pueblos originarios, por el reconocimiento de sus saberes ancestrales, prácticas y cuido a la Madre Tierra, profesionalizar talentos humanos para que armonicen con el Buen Vivir, buscar el desarrollo socio productivo, lograr que dentro de la educación se combine el conocimiento occidental con el ancestral desde los pueblos originarios y así poder aportar en salud, educación, lengua, agricultura, idioma, investigación, entre otros.

Se enfatiza también desde las universidades con la filosofía intercultural y comunitaria en el acompañamiento a los pueblos originarios en procesos de gestión e incidencia para la construcción de ciudadanías que abonen al Buen Vivir, el rol activo de los actores locales en los procesos de investigación e innovación, de esta manera lograr la identidad de los pueblos. Retos importantes, impactantes y trasformadores para las universidades, pueblos originarios, llenos de sabiduría desde las IES.

\section{Desarrollo}

\section{Contexto de la Educación Superior, pueblos Indígenas y afrodescendientes en países de América Latina}

Partamos de recordar la historia de América caracterizada por la conquista y colonización europea, con aniquilaciones, despojos de territorio, desplazamientos, reorganización social y territorial de los pobladores originarios. Los pueblos indígenas americanos y los africanos traídos a América en condiciones de esclavitud, vivieron situaciones que violentaron los elementos de sus visiones de mundo; perdieron sus religiones, los obligaron a usar sus creencias y adoptar una sola religión; el catolicismo.

No se puede recoger la historia sin dejar de apuntar la prohibición del uso de sus lenguas en lugares públicos erradicando su historia, su forma de comunicarse, pues se les indujo a usar un nuevo idioma a conveniencia de los colonizadores, los que tenían el poder. No menos importante es mencionar los conocimientos ancestrales de los pueblos, principalmente en salud, donde ellos practicaban la medicina tradicional que para estos pueblos era la viva curación de enfermedades, pero los colonizadores la satanizaron tachándola de brujería, incluso eran sentenciados a la horca si los encontraban ejerciéndola, término que hoy en día todavía se usa para aquellos indígenas que practican la medicina tradicional.

Existen en pleno siglo XXI normas, leyes, decretos, convenios que abogan por el reconocimiento a la cosmovisión de los pueblos indígenas y afrodescendientes, sus derechos a la educación, territorialidad, gobernanza, idioma, respetar sus creencias, rescatar su producción de conocimiento, tradiciones, diversidad cultural; aunque existen instituciones, Estados, gobiernos, que continúan separando a estos pueblos y negando todo valor que trae consigo cada cultura, cada pueblo, que vive en nuestra América Latina; así lo afirma Mato (2018):

Un aspecto importante de la problemática político-social, educativa y epistemológica contemporánea, es que aún hoy, en pleno Siglo XXI, esta historia de prohibiciones, exclusiones y subordinaciones no ha acabado, sino que continúa bajo diversas formas en la escuela y en las universidades y otras Instituciones de Educación Superior (IES) convencionales (p.18). 
También, podemos mencionar el Convenio 169 de la Organización Internacional del Trabajo (OIT) en 1989. Es el principal instrumento internacional sobre derechos de los pueblos indígenas que en el artículo 5 cita lo siguiente; $\mathrm{Al}$ aplicar las disposiciones del presente convenio:

a) Deberán reconocerse y protegerse los valores y prácticas sociales, culturales, religiosos y espirituales propios de dichos pueblos y deberá tomarse debidamente en consideración la índole de los problemas que se les plantean tanto colectiva como individualmente;

b) Deberá respetarse la integridad de los valores, prácticas e instituciones de esos pueblos;

c) Deberán adoptarse, con la participación y cooperación de los pueblos interesados, medidas encaminadas a allanar las dificultades que experimenten dichos pueblos al afrontar nuevas condiciones de vida y de trabajo.

Fue a través de este convenio 169 que muchos pueblos han reivindicado sus derechos a la educación, salud, idioma, creencias, culturas, conocimientos ancestrales, además ha beneficiado a las universidades comunitarias interculturales para que se organicen y puedan estimular el cumplimiento de este convenio, lograr que luchen por una educación horizontal, de igualdad para todos, sin discriminación, reconociendo los conocimientos de los pueblos, su idiosincrasia, su organización, sus propias formas de aprendizaje, estableciendo la enseñanza desde sus propios currículos que sean inclusivos, donde participen el académico y no académico (sabios, sabias). Incluso este convenio que fue firmado por 15 países de América Latina, y ratificado por 14 paises, ha dado paso a incluir en algunas constituciones políticas de estos países artículos que benefician y reconocen que existen dichos pueblos, que son meritorios de reconocer sus derechos.

Desde la segunda Conferencia Regional de Educación Superior, realizada en Cartagena de Indias en 2008 (CRES 2008), en su declaración final incluyó "los pueblos indígenas y afrodescendientes han tenido más acceso a la formación en educación superior y se han desarrollado respuestas innovadoras en aspectos de calidad, pertinencia y relevancia" (Mato, 2018, p. 8). Este compromiso debe incluir a los docentes, investigadores, empleados y autoridades, también a los que están encargados de la formación profesional, científica y humanística. Solo si a ellos se les sensibiliza sobre la relevancia de comprender un sistema inclusivo, horizontal, de reivindicación de derechos y que la educación no debe hacer distinción alguna con las personas que pertenecen a los pueblos indígenas, se alcanzaría el sueño que la CRES en su tercera conferencia ha planteado, aunque no se puede negar que se han dado pasos sustanciales en temáticas de inclusión, derechos, interculturalidad, entre otros; los que han sido atendidos por universidades que defienden estas acciones.

La deuda histórica de los Estados, universidades se ha minimizado porque actualmente se están impulsado normas, leyes para incluir en la educación, promover los derechos universales a estos pueblos, pero existe un déficit en la aplicación efectiva de los mismos, en donde se escriben convenios, decretos, leyes y no se logran cumplir a cabalidad. Existen países que han venido incluyendo dentro de sus normas, leyes y constituciones políticas la inclusión, derecho, reconocimiento de los pueblos indígenas y afrodescendientes, relevantes para la visibilización, rescate de su cosmovisión, idioma, territorio, y más.

\section{Así es el caso de Bolivia que dentro de sus leyes creó la Ley 018 en junio del 2010, que cita en su artículo 8:}

Consiste en la aplicación obligatoria de la paridad y alternancia en la elección y designación de todas las autoridades y representantes del Estado, en la elección, designación y nominación de 
autoridades, candidaturas y representantes de las naciones y pueblos indígenas originarios campesinos mediante normas y procedimientos propios (Choque, 2018, p. 64).

Sólo con esta ley se denota que hay una aceptación y disposición del Estado en aceptar a los indígenas preparados profesionalmente a optar a cargos públicos del Estado Plurinacional, aunque no podemos olvidar en la historia de Bolivia que desde 1905 se dieron los primeros pasos en la educación para los indígenas, desde entonces se comenzaron a impulsar más escuelas para el acceso a los indígenas, lamentablemente eran escuelas con la visión colonizadora donde se perdía la cultura, las tradiciones, la historia y todo cuanto rodea a la identidad.

Argentina, un país que tenía política de blanqueamiento, es decir no se reconocía al indígena, sino que apuntaban al mestizaje (blanco) como símbolo de poder, haciendo a un lado a los pueblos indígenas y aún más a los afrodescendientes, pero ya hace más de veinte años se han impulsado luchas desde los pueblos indígenas y afrodescendientes para lograr el reconocimiento y gozar así de derechos. Logran dar un giro a la agenda pública, en donde se crea el Instituto Nacional de Asuntos Indígenas (INAI, en 1991), otra ley nacional importante fue la 24.071, en el año 2000 donde se adopta el Convenio 169 (OIT), además con la promulgación de la Ley de Educación Nacional se creó la Modalidad de Educación Intercultural Bilingüe y, posteriormente se conformó el Consejo Educativo Autónomo de Pueblos Indígenas (CEAPI) y pues hay muchas instituciones que están atendiendo el caso de los pueblos, pero no se han dado pasos sustanciales para la educación superior, el mismo hecho de no crear universidades interculturales.

Mientras que, en Brasil, país con una población mayoritariamente afrodescendiente, también da pasos para el reconocimiento de estos pueblos, pero es hasta finales del siglo XX, en su historia relata que era un país que promovía la esclavitud de negros, y no se reconocía como un pueblo con origen, historia, tradiciones, idioma, conocimiento, diversidad cultural, sino que eran invisibilizados, había desigualdad en la distribución territorial. La lucha es fundamental a favor de la población negra, que debería responder a la diversidad étnico-racial al atender en las universidades públicas y privada a afrodescendientes e indígenas.

En Centroamérica; Nicaragua, Costa Rica, Honduras, Guatemala, ratificaron el convenio 169 (OIT), el que da la pauta para que se reconozcan los derechos de los pueblos indígenas y afrodescendiente, específicamente en Nicaragua el Estado ha decretado la Ley No. 28 "Estatuto de Autonomía de las Regiones de la Costa Atlántica de Nicaragua”, publicada el 30 de octubre de 1987. El presente Estatuto establece el Régimen de Autonomía de las Regiones en donde habitan las comunidades de la Costa Atlántica de Nicaragua, reconoce los derechos y deberes propios que corresponden a sus habitantes (creol, miskitos, mayagnas, rama, garífunas) de conformidad con la Constitución Política, da autonomía a los pueblos sobre su cultura, reconoce que es de naturaleza multiétnica, mantener las formas comunales de propiedad de sus tierras, administración, gobernanza, educación, salud, para fortalecer desde su cosmovisión, rescate de sus valores ancestrales, lengua y tradiciones, pero además gozan las Regiones Autónomas de una partida presupuestaria que abona a mejorar la educación, salud, sociedad.

Existen más Estados como Colombia, Ecuador, Chile, México, Perú, entre otros, que han realizado esfuerzos por incluir dentro de sus políticas, leyes, y normas que incluyen el reconocimiento de los pueblos indígenas y afrodescendientes; el pequeño desfase es que en su mayoría estas políticas quedan solo escritas y no pasan al accionar, es allí que se incumplen los acuerdos y es por ello que se sigue promoviendo desde las redes de universidades comunitarias interculturales, organizaciones que apoyen estas iniciativas a seguir impulsando la concretización de las mismas para gozar de los derechos como pueblos. 


\section{i. Educación Superior, pueblos indígenas y afrodescendientes en América Latina: El reto de las Universidades comunitarias interculturales}

Desde las dos últimas décadas han surgido las universidades indígenas, para equilibrar a las universidades convencionales contemporáneas, estas últimas con una visión de educación colonizadora, donde se promueve la inequidad, desigualdad, cupos en fracción para becas a personas indígenas y afrodescendientes, está claro que estas universidades abonaban a la educación de los que tienen poder. Las Universidades o Instituciones de Educación Superior dirigidas para rescatar los conocimientos ancestrales, educación, idioma, diversidad cultural, tradiciones, cosmovisión, son las encargadas de velar porque se cumplan los convenios, leyes, normas existentes en Latinoamérica para beneficio de los pueblos originarios. Al reflexionar sobre estos pueblos existentes que se señalan como la minoría, muchas veces se relaciona a inequidades cultural, moral, económica, social y profesional, por ende, las universidades interculturales y comunitarias nacen para impulsar, fortalecer y determinar el posicionamiento académico con identidad de los pueblos y la construcción de una nueva sociedad.

Es así que estas Instituciones de Educación Superior (IES) se están organizando en redes importantes para lograr la revitalización de los derechos de estos pueblos, en conjunto con académicos y no académicos que buscan cumplir un sueño en beneficio de pueblos originarios. Las universidades interculturales comunitarias están en su apogeo en el siglo XXI, se dinamizan en tomar espacios sustanciales y de toma de decisiones para impulsar el reconocimiento de la diversidad cultural existente en Latinoamérica, los conocimientos endógenos, idioma, cultura, entre otras virtudes que poseen en su interior dichos pueblos.

En la declaración de la Conferencia Regional de Educación Superior (CRES, 2018), se afirma que en manos de las universidades están los desafíos y retos que debemos enfrentar, son de tal magnitud que, de no ser atendidos con oportunidad y eficacia, ahondarán las diferencias, desigualdades y contradicciones que hoy impiden el crecimiento de América Latina y el Caribe con equidad, justicia, sustentabilidad y democracia para la mayoría de los países que la conforman. Esta Conferencia Regional señala que, si bien se ha avanzado hacia una sociedad que busca cambios y referentes democráticos y sustentables, aún faltan transformaciones profundas en los ejes que dinamizarán el desarrollo de la región, entre los cuales, uno de los más importantes, es la educación y en particular la Educación Superior.

En la CRES 2018, los desafíos proyectados son en cuanto a la investigación, la calidad, la extensión y la innovación como motores del desarrollo humano, social y económico en Latinoamérica y el Caribe, donde este mundo nos sumerge; se debe estar preparado para enfrentarlo, ya que se avanza rápidamente en las investigaciones y diferentes paradigmas, respondiendo a las exigencias e interrogantes de este siglo. La tecnología y la innovación son elementos que en el actual contexto toman auge porque están centradas en el desarrollo a nivel internacional desde los avances tecnológicos y se han creado proyectos de innovación donde los conceptos pasan a la práctica y eso es innovar para dar respuesta a los diferentes desafíos que nos engloban.

Las IES tienen grandes retos para una educación intercultural, como es la generación de conocimientos, formación de profesionales integrales, competentes y con valores, ofertas más diversificadas y vinculadas a la investigación, fortalecimiento del proceso de internacionalización, desarrollo de las tecnologías de la información, conocimiento de saberes, identidad intercultural y del sistema de evaluación y participación de la comunidad universitaria en la gestión institucional. Hace dos décadas se están dando pasos para pasar de la educación convencional a un diálogo de saberes a esa educación inclusiva con igualdad de derechos, con pertinencia, atendiendo a la diversidad de forma incluyente, tomando en cuenta los contextos actuales y las demandas de los pueblos, se debe seguir visionando la transformación en los currículos donde se incluyan 
los conocimientos endógenos, reconocimiento de saberes, producción de conocimientos y prácticas desde la cosmovisión de los pueblos originarios.

\section{Existen experiencias de universidades comunitarias interculturales en Latinoamérica como:}

\section{Universidad de las Regiones Autónomas de la Costa Caribe Nicaragüense (URACCAN)}

El Estado de Nicaragua en la Constitución Política, título VII, Artículo 121, reconoce la educación libre en igualdad de condiciones para todos y todas, enfatiza que los pueblos indígenas y las comunidades étnicas de la Costa Atlántica tienen derecho a la educación intercultural en su lengua materna, tampoco se puede dejar de mencionar el marco jurídico legal, en donde existe una Ley de Autonomía de las Instituciones de Educación Superior (Ley No.89) con sus reformas en la asignación, el presupuesto universitario a las dos universidades de la Región: URACCAN y BICU, también se cuenta con la Ley No.218, aprobada en 1996, en su artículo 1, enfatiza en el reconocimiento de las universidades del Caribe de Nicaragua, haciéndoles partícipes de la distribución del 6\% anual del Presupuesto Nacional de la República (Hooker, 2017).

Es así que en 1992 se funda laURACCAN, una universidad comunitaria intercultural, para dar la oportunidad a talentos humanos que se profesionalicen en cada Región, impulsando la Educación Superior, pues en estas regiones no existían universidades para profesionalizarse, por la distancia, caminos en mal estado, lejanía, falta de becas, por ser pueblos indígenas y afrodescendientes. La URACCAN desde su fundación ha preparado más de 8,09o talentos humanos (Registro académico, 2018) que han venido a transformar, fortalecer, mejorar, impulsar y ser reconocidos el trabajo de profesionalización de cada uno en las dos Regiones Autónomas Sur y Norte de Nicaragua.

Dentro de la filosofía Institucional de URACCAN (2015) tiene como visión:

Ser líder en el modelo de universidad comunitaria intercultural reconocida a nivel nacional e internacional por su calidad y pertinencia, que acompaña procesos de gestión e incidencia para la construcción de ciudadanías interculturales de género y el Buen Vivir de los pueblos indígenas, afrodescendientes, mestizos y comunidades étnicas (URACCAN, 2015, p. 7).

La URACCAN un modelo en educación superior fundamentado en la interculturalidad, diálogos de saberes, el papel de la comunidad, los sabios, ancianos, autoridades tradicionales y no tradicionales en la búsqueda de nuevos paradigmas, enfoques metodológicos y de generación de conocimientos; la espiritualidad indígena, afrodescendiente, mestiza y comunidades étnicas; el rol activo de los actores locales en los procesos de investigación y la innovación; perspectiva intercultural de género; la articulación entre teoría y práctica; educación para todos y todas, para toda la vida y la vinculación e incidencia para el desarrollo con identidad y el Buen Vivir de los pueblos, reconocida como la primera Universidad Comunitaria Intercultural de ABYA YALA (URACCAN, 2015).

Actualmente está inmersa en el proceso de acreditación, la que debe cumplir con estándares de calidad, pertinencia, eficiencia, eficacia, la evaluada con parámetros de universidades convencionales, aunque se han realizado esfuerzos para que los pares evaluadores reconozcan que la URACCAN es una universidad diferente, concebida como comunitaria intercultural.

\section{Universidad Autónoma Indígena Intercultural (UAIIN), Colombia, Cauca}

Creada por el Consejo Regional Indígena del Caribe (CRIC), fundada en 2003, lo relevante es que es una universidad desde/con y para los pueblos indígenas, nace y se fortalece con ellos, existen conflictos directos con las universidades convencionales, con formatos, normas, procesos que engloban este tipo 
de universidad. Palechor (2017), afirma que, "no existe educación superior sin educación inferior, pues el niño desde que está en el vientre de la madre es educado, es un proceso continuo, que se realizada día a día, hasta que pasan a otra dimensión". Está claro que hubieron profesionales que apoyaron a la UAIIN, pero al final los indígenas terminaron por mostrarle el mundo de ellos para que lograran comprender y así enseñar no sólo lo académico sino los conocimientos ancestrales que tiene consigo cada pueblos, como la salud, idioma, derechos territoriales a la igualdad, educación entre otras, y la necesidad de estar en armonía con la Madre Tierra.

Así que se va formando la UAIIN con el sueño de preparar a su gente profesionalmente, donde prevalezca su cultura, conocimientos, idioma, derechos (defensa de territorio indígena, defensa de la Autonomía), una universidad llamada la "Gran Escuela", es allí donde se encuentra la gran experiencia de los pueblos, su sabiduría, organización, cuidando que prevalezca la identidad a nivel general. El campo que prepara esta universidad es salud, derecho, comunicación, pero especialmente en la educación (Palechor, 2017)

Finalmente, destacar que se está en un proceso de acreditación, pero es medida con parámetros estandarizados, desde universidades convencionales, olvidando la particularidad, diversidad cultural que engloba a una universidad intercultural.

\section{Universidad Intercultural de las Nacionalidades y Pueblos Indígenas (AMAWTAY WASI), Ecuador}

Una universidad que actualmente está entrando en debate para ser acreditada, ya que desde el 2013, había sido cerrada por el Gobierno, aunque ha recuperado su posicionamiento, al consultar a Sarango (2009) Amawtay Wasi, nace con la visión:

La recuperación de un tejido vivo que entretejemos en la interculturalidad cósmica. Su misión es contribuir en la formación de talentos humanos que prioricen una relación armónica entre la Madre Naturaleza y el ser humano sustentándose en el bien vivir comunitario como fundamento de la construcción del Estado Plurinacional y la Sociedad Intercultural. (p.3)

Se funda para responder a las necesidades de reconocimiento de sus saberes, conocimientos, prácticas en armonía con la Madre Tierra, basada en formar talentos humanos que armonicen con el Buen Vivir, pero también se logra con esta universidad que se reconozca a la comunidad como sujetos; donde se otorga el reconocimiento del quehacer académico.

\section{Universidades de Bolivia (UNIBOL)}

Se crea por Decreto Supremo en el 2008, tres universidades indígenas bolivianas comunitarias interculturales y productivas, llamadas UNIBOL, distribuidas por contexto cultural, para responder la demanda de los pueblos Quechua, Aymara, Guaraní, dedicadas al rescate de sus culturas, conocimiento ancestral destacando la universidad como espacio trascendental para generar, recrear y socializar conocimiento en convivencia humana. Me enfocaré en la UNIBOL Guaraní que nace con la visión de:

La complementariedad de los saberes y conocimientos ancestrales y científicos en función a las necesidades de desarrollo socio productivo y sociopolítico del Estado Plurinacional; y define su misión institucional como de carácter intracultural, intercultural y plurilingüe, para que respondan con calidad y pertinencia a las demandas... (Mandepora, 2016)

Vela por la formación profesional de los integrantes de la comunidad indígena, pues es desde allí que se cuida por no perder las experiencias, producción de conocimiento, lengua, cultura, conocimientos 
ancestrales. Esta universidad buscaba preparar al recurso humano que se identifique, hable y escriba en su lengua originaria desde sus docentes, esto es solo un sueño, ya que no existen profesionales preparados en las carreras que oferta, por consiguiente, la parte técnica y profesional están en manos de docentes de habla castellano aunque también cuenta con profesionales en educación bilingüe que son indígenas.

Al finalizar esta mirada sobre algunas universidades comunitarias interculturales, se nota el crecimiento, los obstáculos que han sobrepasado, la desigualdad que existen para este tipo de universidad en el sistema educativo convencional; pero, no se puede dejar de destacar la visión y misión por las que surgen cada una de ellas, las que homologan por un mismo fin; donde todos los miembros de sus comunidades indígenas, afrodescendientes, mestizos y otras comunidades étnicas, puedan gozar del acceso a la educación superior, prepararse en ese contexto y mejorar el plan de vida su comunidad y de ellos como personas.

\section{ii. Interculturalidad, colaboración intercultural en investigación: un aporte en conjunto con las comunidades}

Deseo compartir este capítulo haciendo un breve recorrido por el concepto de Interculturalidad desde diferentes visiones, su uso depende en gran medida por los pueblos que la practican, por el contexto donde lo utilizan, es un término que se usa no sólo para la investigación, sino por leyes, normas, empresas, instituciones gubernamentales y no gubernamentales, organizaciones indígenas y afrodescendientes, es un término que está en debate desde las organizaciones sociales que abona a comprender a profundidad la interculturalidad.

Ocoró (2018) enfatiza: "la perspectiva Intercultural se propone como un camino posible para lograr el reconocimiento y el respeto del "otro", un modo de trascender el etnocentrismo y de abrirse a la valoración de otros conocimientos" (p.7).

Para URACCAN, (2016); la interculturalidad es concebida como:

Un proceso permanente de construcción, establecimiento y fortalecimiento de espacios de diálogo, comunicación e interacción horizontal de doble vía, entre personas, comunidades y pueblos 
de diferentes culturas...Su finalidad es la promoción y práctica de la equidad, el respeto, la comprensión, la aceptación mutua y la creación de sinergias para el establecimiento de sociedades inclusivas, no discriminatorias y libres de racismo.

Otra forma de comprender la interculturalidad señalada por Mato (2009); en sus escritos remarca "las relaciones interétnicas entre pueblos o grupos sociales diferenciados por referentes raciales, lingüísticos o de origen nacional" (p.33), también afirma que al consultar extensas bibliografías la idea de interculturalidad para diversos países se centra en "casos relacionados con diferencias étnicas, religiosas, raciales o del país de origen de poblaciones migrantes".

\section{Conclusiones}

Desde las diferentes revisiones bibliográficas, existen más ideas sobre interculturalidad visualizadas desde la sociedad, pueblos originarios, empresas, académicos, educación, gobierno, Estado, actores involucrados, es un término que se sigue debatiendo hasta nuestros días, sus interpretaciones dependen de cada agente social que la utiliza en un mismo contexto o en contextos diferentes, en prácticas interculturales, producción de conocimiento desde los pueblos, en el marco del respeto de ideologías. Puedo afirmar que este término sigue en movimiento, en construcción. Es muy polémico y su interpretación como afirmé anteriormente depende del contexto.

Un desafío es que las universidades los acompañen, esto implica capacitar a los académicos, investigadores, estudiantes que se identifiquen, sientan, vivan la necesidad de recuperar a través de la investigación los conocimientos endógenos para ser reconocidos científicamente. Es necesario mencionar que para hacer investigación y que sea válida existen formatos, procesos, paradigmas. Es allí que como universidades comunitarias interculturales luchan por el reconocimiento de su nueva forma de enseñar y aprender, remarcando que no son iguales a las universidades convencionales y que desde esta filosofía se pueden hacer investigaciones con nuevos paradigmas como la investigación acción participativa, colectiva donde la comunidad es sujeto y protagonista de la investigación vinculada, una investigación realizado desde/ con/para la comunidad, una relación horizontal (universidad-comunidad), donde se deje evidencia de conocimiento y haceres de los pueblos.

El reto es hacer evidente el diálogo de saberes, salir de espacios cerrados como las aulas de clase, trasladarse a los espacios vivos para aprender en los lugares apropiados en armonía con la Madre Tierra, sentir, vivir, convivir con los diferentes pueblos indígenas y afrodescendientes, donde se puedan identificar en conjunto algunas problemáticas y se puedan buscar soluciones que beneficien a la comunidad, siempre y cuando haya disponibilidad.

\section{Lista de referencias}

CRES (2018). Córdoba: Carta de las Universidades e Instituciones de Educación de los Pueblos Indígenas a la Disponible en: http://www.cres2018.org/uploads/carta_pueblos_originarios.pdf

Convenio Núm. 169 de la OIT sobre Pueblos indígenas y tribales (20014): Declaración de las Naciones Unidas sobre los Derechos de los pueblos indígenas.

Hernández Loeza, Sergio Enrique (2018). Educación superior, diversidad cultural e interculturalidad en América Latina. Estudio sobre México. En: Daniel Mato, coord. 
Hooker Blanford, Alta (2017). Avances y desafíos en la evaluación de la calidad de universidades y programas para y con pueblos indígenas y afrodescendientes. Educación Superior y Sociedad. 20 (número): $183-210$.

Hooker Blanford, Alta (2018). Universidades e instituciones de educación superior indígenas, interculturales, afrodescendientes y comunitarias en América Latina. En: Daniel Mato, coord., Educación Superior, Diversidad Cultural e Interculturalidad en América Latina.

Mato, Daniel (2008). No hay saber "universal”, la colaboración intercultural es imprescindible. Alteridades 18(35):101-116.

Mato, Daniel (2009). Contextos, conceptualizaciones y usos de la idea de interculturalidad. En: Miguel Ángel Aguilar y otros, coords. Pensar lo contemporáneo: De la cultura situada a la convergencia tecnológica. Barcelona y México: Anthropos Editorial y Universidad Autónoma Metropolitana-Iztapalapa.

Mato, Daniel (2014). Universidades Indígenas en América Latina. Experiencias, logros, problemas, conflictos y desafíos. Inclusión Social y Equidad en la Educación Superior (Revista ISEES).14: 17-45.

Mato, Daniel (2016). Educación Superior y Pueblos Indígenas en América Latina: Del “Diálogo de Saberes" a la construcción de modalidades sostenibles de "Colaboración Intercultural". Revista del CISEN Tramas/Maepova 4(2): 71-94.

Mato, Daniel (2018). Educación Superior y Pueblos Indígenas y Afrodescendientes en América Latina. Aspectos sociales y normativos, experiencias, logros, aprendizajes y desafíos. En: Daniel Mato, coord. Educación Superior, Diversidad Cultural e Interculturalidad. Caracas: UNESCO-IESALC y Córdoba: Universidad Nacional de Córdoba.

Mato, Daniel (2018). Educación Superior y Pueblos Indígenas y Afrodescendientes en América Latina: Tendencias en curso, tensiones, posibilidades y desafíos. En: Pedro Henríquez Guajardo (coord.) Tendencias de la educación superior en América Latina y el Caribe 2018-2028 Caracas: UNESCO - IESALC y Córdoba: Universidad Nacional de Córdoba (ISBN: 978-980-7175-34-0).

Mazabel Cuásquer, Milena (2018). Educación superior, diversidad cultural e interculturalidad en Colombia. En: Daniel Mato, coord. Educación Superior, Diversidad Cultural e Interculturalidad.

Mora Curriao, Maribel (2018). Educación superior, diversidad cultural e interculturalidad en Chile. En: Daniel Mato, coord. Educación Superior, Diversidad Cultural e Interculturalidad.

Olivera Rodríguez, Inés (2018). Educación superior y pueblos indígenas y afrodescendientes en el Perú: avances $y$ desafíos en el marco actual de las políticas. En: Daniel Mato, coord. Educación Superior, Diversidad Cultural e Interculturalidad.

Palechor Arévalo, Libio (2017). La Universidad Autónoma Indígena Intercultural: una apuesta a la construcción de interculturalidad. Educación Superior y Sociedad. 20, 157-182.

Tuaza Castro. Luis Alberto (2018). Educación superior y pueblos indígenas y afrodescendientes en Ecuador En: Daniel Mato, coord. Educación Superior, Diversidad Cultural e Interculturalidad. 
URACCAN, (2016). Proyecto Educativo Institucional de la Universidad de las Regiones Autónomas de la Costa Caribe Nicaragüense. Managua: URACCAN.

URACCAN, (2015). Plan estratégico de la Universidad de las Regiones Autónomas de la Costa Caribe Nicaragüense. Managua: URACCAN.

Zúñiga Muñoz, Xinia (2018). Persistencias coloniales, aperturas y desafíos para la educación superior en Centroamérica. En: Daniel Mato, coord. Educación Superior, Diversidad Cultural e Interculturalidad. 117-142. 